\title{
Cavitating left upper lobe pneumonia; a case of Melioidosis
}

\author{
Kannangara LS ${ }^{1}$, Samarasekara GBL ${ }^{1}$, Kularatne $\mathbf{W N S}^{1}$, Corea $\mathbf{E}^{2}$, Elvitigala $\mathbf{J}^{\mathbf{3}}$, Masakorala $\mathbf{J}^{\mathbf{2}}$ \\ ${ }^{\prime}$ National Hospital for Respiratory Diseases, Sri Lanka \\ ${ }^{2}$ Department of Microbiology, Faculty of Medicine, University of Colombo, Sri Lanka \\ ${ }^{3}$ National Tuberculosis Laboratory, Sri Lanka
}

Correspondence: Dr. L.S. Kannangara

\begin{abstract}
Melioidosis is a bacterial infection caused by Burkholderia pseudomallei. The first cases of melioidosis were described in Burma just over a century ago. Since then, the endemic zone has expanded and includes Sri Lanka. The clinical presentation of melioidosis ranges from acute, subacute and chronic manifestations. Due to its protean clinical presentation, a high index of suspicion is necessary for the clinical diagnosis. Diagnosis is confirmed by isolation of B. pseudomallei from clinical specimens. A high or rising antibody titre to melioidin antigen is supportive, but not diagnostic. B. pseudomallei grows readily in commonly used laboratory media but may not be identified unless laboratory personnel have prior experience with this organism. Treatment is complex and includes a prolonged course of intravenous antibiotics followed by months of oral therapy to ensure eradication of the bacterium. Relapse is common in spite of adequate therapy. A case report of a patient with acute onset pneumonia with a positive sputum culture of B.pseudomallei is presented.
\end{abstract}

\section{Introduction}

Melioidosis is a bacterial infection caused by B.pseudomallei, an aerobic, Gram-negative, motile bacillus, endemic in the tropical and subtropical regions of South East Asia and Northern Australia. Even though Sri Lanka has been considered as nonendemic for melioidosis in the past, it is now recognised as an emerging infection in the country. The first cases of melioidosis were documented in Burma by Alfred Whitmore and C.S. Krishnaswami in 1912 and the first published report of melioidosis in Sri Lanka was in 1927 (1), in a European tea broker residing in Sri Lanka. Since then several case reports of melioidosis acquired in Sri Lanka have been published (2-4).

Melioidosis is acquired by inoculation or inhalation of soil and water contaminated with the bacterium. Occupational exposure to mud and surface water is a risk factor $(5,6)$. More severe disease is seen among patients with diabetes, chronic kidney disease or liver cell disease. Once the organism enters the body, clinical presentation is varied ranging from acute, sub-acute to chronic (6). It resembles the clinical manifestations of tuberculosis and may be misdiagnosed as such. In countries where both infections are prevalent, clinicians need a high index of suspicion to diagnose melioidosis.

Once the diagnosis is made, melioidosis is treated by intravenous (IV) meropenem or imipenem for a minimum duration of two weeks (7). In severe cases IV therapy needs to be prolonged for four to six weeks. Once the initiation phase is over, two drugs, from cotrimoxazole, co-amoxyclav and doxycycline, should be given for at least three months to prevent a relapse. It is recommended that the latter part of the induction phase should overlap with the commencement of cotrimoxazole (7).

\section{Case Report}

A 25-year-old, previously healthy insurance sales executive from Negombo in the Western Province of Sri Lanka presented to a local hospital with a history of fever with chills and rigors for 4 days and productive cough with intermittent haemoptysis, 
loss of appetite, evening pyrexia, and night sweats of two weeks duration. Initial examination at the local hospital was unremarkable except for an elevated temperature. Random blood sugar and fasting blood sugar performed were persistently high and insulin was commenced. His full blood count revealed neutrophil leucocytosis and his ESR and CRP were high. His initial chest radiograph showed apical shadowing on the left side (Figure 1).

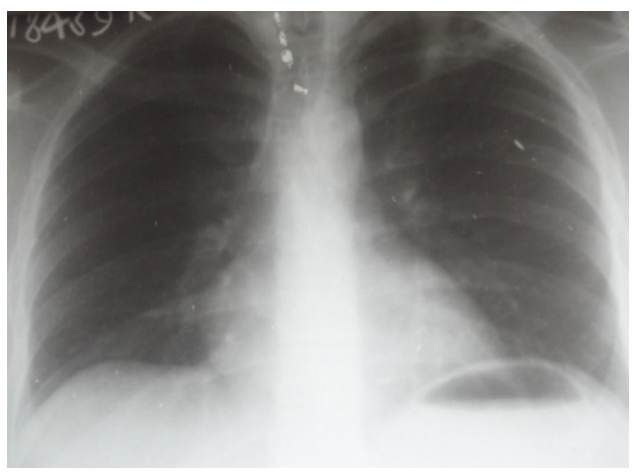

Figure 1: Chest radiograph showing early left apical shadow

He was started on intravenous cefuroxime and discharged on oral antibiotics once the fever settled. However he was readmitted to the local hospital three days later due to the recurrence of fever. On examination he was febrile, ill looking and examination of the respiratory system revealed a patch of bronchial breathing in the left upper zone. The white cell count and the rising CRP and ESR were suggestive of an unresolving bacterial infection. Repeat chest radiograph revealed left upper zone opacity with cavitation (Figure 2).

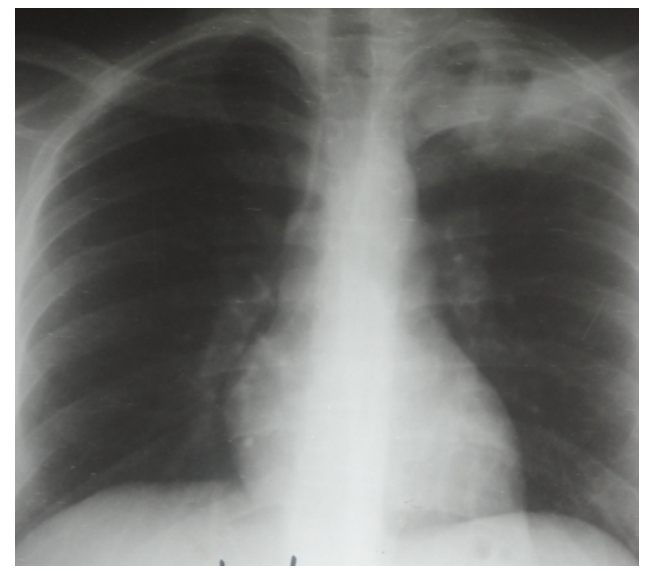

Figure 2: Chest radiograph showing left upper zone opacity with cavity formation
He was started on intravenous meropenem $1 \mathrm{~g}$ 8 hourly after taking sputum and blood for culture and transferred to the National Hospital for Respiratory Diseases (NHRD), Welisara for further management.

On admission to the NHRD, clinical examination revealed an ill looking, febrile patient with a patch of bronchial breathing and crepitations over the left upper zone of the lung fields. There was no evidence of pleural effusion, a cardiac murmur or organomegaly. Three specimens of sputum were negative for acid fast bacilli (AFB) by microscopy and culture and the Mantoux test was negative. Ultrasound imaging of the abdomen revealed no abnormality. Sputum for pyogenic culture grew a bacterium that was identified as a pseudomonas species suggestive of B.pseudomallei. Antibody to melioidin by the indirect haemagglutination test (IHA) was positive at a titre of 1:2560 and the diagnosis of melioidosis was made. The isolate was subsequently confirmed to be $B$. pseudomallei by matrix-assisted laser desorption / ionization time of flight mass spectrometry (MALDI-TOF) and PCR at the reference laboratory.

Intravenous meropenem was continued and oral cotrimoxazole $1920 \mathrm{mg}$ twice daily was added after one week. Fever settled after one week of therapy. He was discharged on oral cotrimoxazole and doxycycline $100 \mathrm{mg}$ twice daily after completing 2 weeks of intravenous therapy. On review, 2 weeks after discharge, he was clinically well with good glycaemic control. His inflammatory markers were normal and a repeat chest radiograph showed resolution of the lung lesion (Figure 3). He is currently on oral cotrimoxazole and doxycycline for a total of three months.

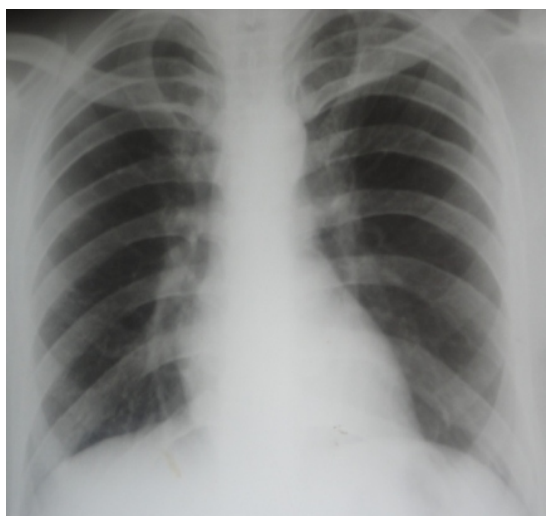

Figure 3: Chest radiograph showing resolved opacity

Galle Medical Journal, Vol 19: No. 1, March 2014 


\section{Discussion}

Melioidosis is an emerging infection in Sri Lanka and an increasing number of cases are being reported, probably due to improvement in clinical awareness and the availability of clinical microbiology laboratory facilities. Clinical presentation, similar to tuberculosis, is varied, ranging from acute to chronic or even sub acute, as in our patient.

Our patient was a recently diagnosed diabetic with a sub acute presentation of an unresolving pneumonia with cavity formation. This triad of clinical features is typical of tuberculosis which resulted in his transfer to the NHRD for further investigation. However, both sputum microscopy and culture were negative for acid fast bacilli as was the skin test for evidence of cell mediated immunity to tuberculosis. Pyogenic culture, on the other hand, revealed a pure growth of a Pseudomonas-like bacterium. Due to a high index of suspicion by the clinician and prior experience with B. pseudomallei by the microbiologist, melioidosis was suspected and the isolate sent to the reference laboratory for further identification (2).

The initial identification was made due to the typical colony appearance on blood agar (8) i and MacConkey agar. On blood agar, colonies were pinpoint at 24 hours. After 48 hours opaque, creamy white colonies with beta haemolysis were seen (Figure 4). Over the next week the colonies became umbonate (raised centre) and then developed wrinkling. On MacConkey agar colonies had a nonlactose fermenting appearance at 24 hours but by 48 hours had turned the bright pink colour of a lactose fermenter (Figure 5). However it is important to note that a minority of strains show translucent, serous grey colonies with only limited haemolysis on blood agar and have a non-lactose fermenting (NLF) appearance on MacConkey agar.

The isolate had the characteristic earthy smell described for B. pseudomallei, typical "safety pin" appearance on Gram stain, a positive oxidase test and gentamicin, polymyxin and colistin resistance coupled with co-amoxyclav sensitivity (2) in the antibiotic sensitivity test (ABST) (Figure 6). Vancomycin was included in the ABST to exclude the possibility of a spore bearer which may also give rise to wrinkled colonies with a safety pin appearance on gram stain. However the spore bearer
(Bacillus spp) will be vancomycin sensitive while Burkholderia spp are vancomycin resistant.

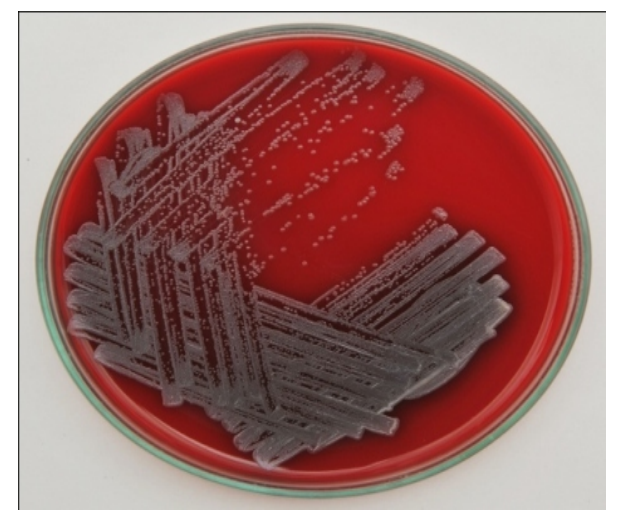

Figure 4: Pinpoint colonies with white opaque appearance in confluent areasance seen on blood agar

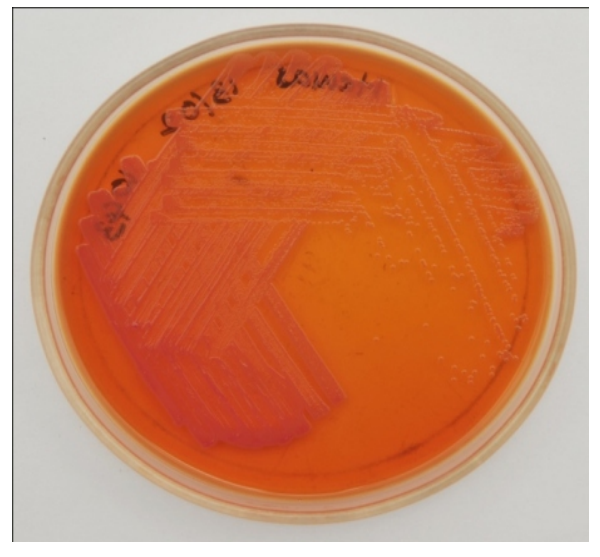

Figure 5: Pink colonies on-MacConkey agar

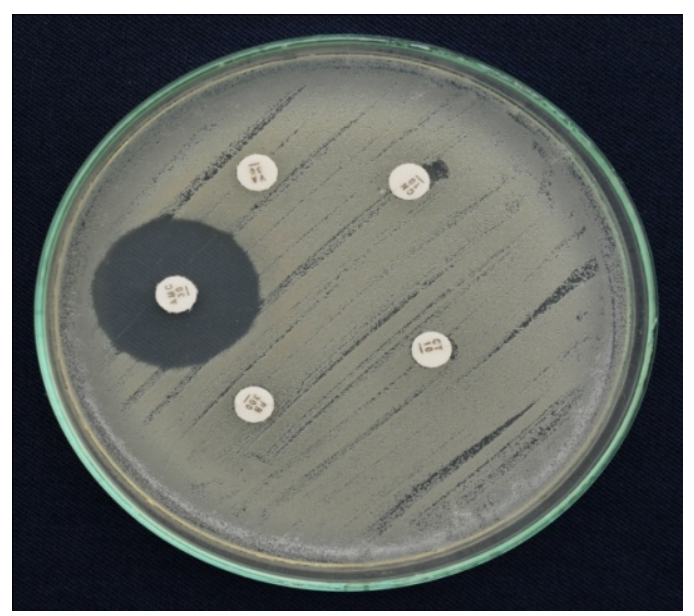

Figure 6: Antibiotic sensitivity pattern with gentamicin, polymyxin and colistin and vancomycin (resistance coupled with coamoxyclav sensitivity 
Confirmation of identity required real time PCR and MALDI-TOF mass spectrometry which were not available locally and were performed at the reference laboratory (9).

Effective treatment of the patient and a successful outcome was the result of a high degree of clinical awareness of melioidosis and prior experience with B.pseudomallei. It is very likely that melioidosis is more prevalent in Sri Lanka than is currently recognised (2). Many sub-acute and chronic presentations, especially cavitating pneumonia and psoas abscess may be misdiagnosed and treated as tuberculosis, with a fatal outcome. Even if B.pseudomallei is isolated in sputum culture it may not be recognised by laboratory personnel due to lack of prior experience. Even in our patient the initial sputum culture was reported as Pseudomonas spp but awareness on the part of the clinician and microbiologist led to further investigation.

\section{Conclusions}

Melioidosis is a difficult infection to manage, not least because of its capacity to cause a rapidly fatal outcome despite the use of antibiotics. As melioidosis and tuberculosis have similar risk factors and clinical presentations, as in this case, and are prevalent in the same tropical belt, there may be diagnostic difficulties unless a high index of suspicion is maintained. Therefore it is important to raise awareness among clinicians regarding the prevalence and varied clinical presentation of this infection. It is also important to improve bacterial culture facilities and educate laboratory personnel in order to improve the laboratory diagnosis of this infection, especially in laboratories that do not have an on-site clinical microbiologist.

\section{References}

1. Denny CR. Melioidosis in a European. Ceylon Journal of Science 1927; 2: 37-40.

2. E. Corea, V. Thevanasam, S Perera, I Jayasinghe, A Ekanayake, J Masakorala, TJJ Inglis. Melioidosis in Sri Lanka: an emerging infection. Sri Lanka Journal of Infectious Disease 2012; 2: 2-8.

3. Peetermans WE, Van Wjngaerden E, Van Eldere J, Verhaegen J. Melioidosis brain and lung abscess after travel to Sri Lanka. Clinical Infectious Diseases 1999; 28: 921-2.

4. Jayasekara K, Perera S, Wijesundere A. Fatal Burkholderia pseudomallei septicaemia. Ceylon Medical Journal 2006; 51: 69-70.

5. Leelarasamee A, Bovornkitti S. Melioidosis: Review and update. Reviews of Infectious Diseases 1989; 2: 413-25.

6. White NJ. Melioidosis. Lancet. 2003; 361: 1715-22.

7. Timothy JJ Inglis. The Treatment of Melioidosis Pharmaceuticals 2010; 3: 1296-303.

8. Cheng AC, Currie BI. Melioidosis: Epidemiology, Pathophysiology and Management. Clinical Microbiology Reviews 2005; 18: 383-416. Doi:10.1128/CMR.18.2.383416.2005 .

9. GND Perera, LD Dias, A Kulatunga, E Corea, J Masakorala. A Case Report of Melioidosis. Sri Lanka Journal of Infectious Diseases 2012; 1(2): 47-51. 